\title{
One Functional Class of Uniform Convergence on a Segment of Truncated Whittaker Cardinal Functions
}

\section{A.YU. TRYNIN}

Mechanics and Mathematics Faculty, Saratov State University, 83, Astrakhanskaya Street, 410012, Saratov, Russia

ABSTRACT
One functional class is described in terms of one-sided modulus of continuity and the modulus of positive (nega-
tive) variation on which there is a uniform convergence of the truncated cardinal Whittaker functions.
Keywords: Sinc Approximation; Truncated Cardinal Function; Interpolation Functions; Uniform Approximation

\section{Introduction and Preliminaries}

E. Borel and E.T. Whittaker introduced the notion of a truncated cardinal function, whose restriction on the segment $[0, \pi]$ reads as follows:

$$
\begin{aligned}
& \mathrm{C}_{\Omega}(\mathrm{f}, \mathrm{x})=\sum_{\mathrm{k}=0}^{\mathrm{n}} \frac{\sin (\Omega \mathrm{x}-\mathrm{k} \pi)}{\Omega \mathrm{x}-\mathrm{k} \pi} \mathrm{f}\left(\frac{\mathrm{k} \pi}{\Omega}\right) \\
& =\sum_{\mathrm{k}=0}^{\mathrm{n}} \frac{(-1)^{\mathrm{k}} \sin \Omega \mathrm{x}}{\Omega \mathrm{x}-\mathrm{k} \pi} \mathrm{f}\left(\frac{\mathrm{k} \pi}{\Omega}\right),
\end{aligned}
$$

here $\Omega>0$ and $\mathrm{n}=[\Omega]$ is integer part $\Omega \in \mathrm{R}$. The function $\frac{\sin (\Omega \mathrm{x})}{\Omega \mathrm{x}}$ called sinc-function. Up to now, a fairly well-studied problem is the one concerning sinc approximations of an analytic function on the real axis decreasing exponentially at infinity. The most complete survey of the results obtained in this direction by 1993 be found in ${ }^{[1]}$.

Sinc approximations have wide applications in mathematical physics, in constructing various numerical methods and the approximation theory for the functions of both one and several variables ${ }^{[1-7]}$, in theory of quadrature formulae ${ }^{[1,8]}$, in theory of wavelets or wavelet-transforms in ${ }^{[2,9-11]}$. In book ${ }^{[16]}$ designated perspective directions of development of sinc approximations.

One test for the uniform convergence on the axis for Whitteker cardinal functions were provided in ${ }^{[12,13]}$. Another important sufficient condition for convergence of sinc approximations was obtained $i^{[14]}$. It was established that for some subclasses of functions absolutely continuous together with their derivatives on the interval $(0, \pi)$ and having a bounded variation on the whole axis R Kotel'nikov series (or cardinal Whitteker functions) converge uniformly inside the interval $(0, \pi)$. $\operatorname{In}^{[15]}$ was obtained by an upper bound for the best possible approximations of sincs.

Unfortunately, while approximating continuous functions on a segment by means of (1.1) and many other operators, Gibbs phenomenon arises in the vicinity of the segment end-points, see, for instance ${ }^{[18]} \cdot \operatorname{In}^{[19]}$ and $^{[18]}$ various estimates for the error of approximation of analytic in a circle functions by sine-approximations (1.1) (when $\Omega=n$ ) were obtained. In papers ${ }^{[17]}$ there were obtained estimates for the error of approximations of uniformly continuous and bounded on $\mathrm{R}$ functions by the values of various operators being combinations of sincs.

In paper ${ }^{[19]}$ sharp estimates were established for the functions and Lebesgue constants of operator (1.1) (when $\Omega=n$ ). Works ${ }^{[20,21]}$ were devoted to obtaining necessary and sufficient conditions of pointwise and uniform in interval $(0, \pi)$ convergence of values operators (1.1) (when $\Omega=n$ ) for functions $f \in C[0, \pi]$. $\operatorname{In}^{[22]}$ there was constructed an example of continuous function vanishing at the end-points of the segment $[0, \pi]$ for which the sequence of the values of operators (1.1) (when $\Omega=n$ ) diverges unboundedly everywhere on the interval $(0, \pi)$. Work ${ }^{[23]}$ was denoted to studying approximative properties of interpolation operators constructed by means of solutions to the Cauchy problems with second order differential expressions. Papers ${ }^{[24,25]}$ were devoted to applications of considered in $^{[23]} \mathrm{La}-$ grange-Sturm-Liouville interpolation processes.

Copyright @ 2018 A.YU. TRYNIN

doi: 10.24294/ijmss.v1i3.527

EnPress Publisher LLC.This work is licensed under the Creative Commons Attribution-NonCommercial 4.0 International License (CC BY-NC 4.0).

http://creativecommons.org/licenses/ by/4.0/ 
$\mathrm{In}^{[26]}$ the results of work $^{[23]}$ were applied for studying approximative properties of classical Lagrange interpolation processes with the matrix of interpolation nodes, whose each row consists of zeroes of Jacobi als $P_{n}^{a_{n}, \beta n}$ with the parameters depending on $n$. In the works $^{[27-29]}$ of construction of new operators sinc approximations. They allow you to uniformly approximate any continuous function on the segment.

In the present work we follow the lines of publications ${ }^{[30-40]}$. We functional class is described on which there is a uniform convergence of the truncated cardinal Whittaker functions for in terms of one-sided modulus of continuity and the modulus of positive (negative) variation.

Fix $\rho_{\lambda}=o\left(\frac{\sqrt{\lambda}}{\ln \lambda}\right) \quad$ as $\quad \lambda \rightarrow+\infty$, let $h(\lambda) \in$ $\mathrm{R}$, and for each nonnegative $\lambda$ let $\mathrm{q}_{\lambda}$ be arbitrary function in the ball $V_{\rho_{\lambda}}[0, \pi]$ of radius $\rho_{\lambda}$ in the space of functions with bounded variation vanishing at the origin, so that

$$
\mathrm{V}_{0}^{\pi}\left[\mathrm{q}_{\lambda}\right] \leq \rho_{\lambda}, \quad \rho_{\lambda}=\mathrm{o}\left(\frac{\sqrt{\lambda}}{\ln \lambda}\right)
$$$$
\text { as } \lambda \rightarrow \infty, \quad \mathrm{q}_{\lambda}(0)=0 \text {. }
$$

For a potential $\mathrm{q}_{\lambda} \in \mathrm{V}_{\rho_{\lambda}}[0, \pi]$, where $\lambda \rightarrow+\infty$ the zeros of solution of the Cauchy problem

$$
\left\{\begin{array}{c}
y^{\prime \prime}+\left(\lambda-q_{\lambda}(x)\right) y=0, \\
y(0, \lambda)=1, y^{\prime}(0, \lambda)=h(\lambda),
\end{array}\right.
$$

or, provided that $\mathrm{h}(\lambda) \neq 0$

as

$$
\mathrm{V}_{0}^{\pi}\left[\mathrm{q}_{\lambda}\right] \leq \rho_{\lambda}, \quad \rho_{\lambda}=\mathrm{o}\left(\frac{\sqrt{\lambda}}{\ln \lambda}\right)
$$

$$
\lambda \rightarrow \infty, \quad \mathrm{q}_{\lambda}(0)=0, \lambda \rightarrow \infty, \mathrm{q}_{\lambda}(0)=0, \mathrm{~h}(\lambda) \neq 0,
$$

the zeros of Cauchy problem

$$
\left\{\begin{array}{c}
y^{\prime \prime}+\left(\lambda-q_{\lambda}(x)\right) y=0, \\
y(0, \lambda)=1, y^{\prime}(0, \lambda)=h(\lambda),
\end{array}\right.
$$

which lie in $[0, \pi]$ and are numbered in ascending order, will be denoted by

$$
\begin{gathered}
0 \leq \mathrm{x}_{0,} \lambda<\mathrm{x}_{1}, \lambda<\cdots<\mathrm{x}_{\mathrm{n}(\lambda),} \\
\lambda \leq \pi \quad\left(\mathrm{x}_{-1, \lambda}<0, \mathrm{x}_{\mathrm{n}(\lambda)+1, \lambda}>\pi\right) .
\end{gathered}
$$

(Here $x_{-1, \lambda}<0$, and $x_{n}(\lambda)+1, \lambda>\pi$ are the zeros of the extension of solution of the Cauchy problem (1.3) or (1.5) corresponding to some extension of function $q_{\lambda}$ outside $[0, \pi]$ having similar bounds for the variation).

$\mathrm{In}^{[23]}$ the properties of the Lagrange type approximation investigated. The operators which include the solution of the Cauchy problem of the form (1.5) or (1.6) and the continuous function which bind

$$
\begin{aligned}
& \mathrm{S}_{\lambda}(\mathrm{f}, \mathrm{x}) \\
& =\sum_{\mathrm{k}=0}^{\mathrm{n}} \frac{\mathrm{y}(\mathrm{x}, \lambda)}{\mathrm{y}^{\prime}\left(\mathrm{x}_{\mathrm{k}, \lambda}, \lambda\right)\left(\mathrm{x}-\mathrm{x}_{\mathrm{k}, \lambda}\right)} \mathrm{f}\left(\mathrm{x}_{\mathrm{k}, \lambda}\right) \\
& =\sum_{\mathrm{k}=0}^{\mathrm{n}} \mathrm{S}_{\mathrm{k}, \lambda}(\mathrm{x}) \mathrm{f}\left(\mathrm{x}_{\mathrm{k}, \lambda}\right) ;
\end{aligned}
$$

it interpolates $f$ at the nodes $\left\{\mathrm{x}_{\mathrm{k}, \lambda}\right\}_{\mathrm{k}=0}^{\mathrm{n}}$.

Let $\mathrm{C}_{0}[0, \pi]=\{\mathrm{f}: \mathrm{f} \in \mathrm{C}[0, \pi], \mathrm{f}(0)=\mathrm{f}(\pi)=0\}$.

When approximation using sinc approximations (1.1) function $f \in C[0, \pi] \backslash C_{0}[0, \pi]$ near the endpoints of the Gibbs phenomenon occurs. This problem can be solved with the help of the reception that was used in the construction of the operator ${ }^{[23]}$, formula (1.9)

$\mathrm{T}_{\lambda}(\mathrm{f}, \mathrm{x})$

$$
\begin{aligned}
& =\sum_{\mathrm{k}=0}^{\mathrm{n}} \frac{\mathrm{y}(\mathrm{x}, \lambda)}{\mathrm{y}^{\prime}\left(\mathrm{x}_{\mathrm{k}, \lambda}, \lambda\right)\left(\mathrm{x}-\mathrm{x}_{\mathrm{k}, \lambda,}\right)}\left\{\mathrm{f}\left(\mathrm{x}_{\mathrm{k}, \lambda}\right)-\frac{\mathrm{f}(\pi)-\mathrm{f}(0)}{\pi} \mathrm{x}_{\mathrm{k}, \lambda}-\mathrm{f}(0)\right\} \\
& +\frac{f(\pi)-f(0)}{\pi} x+f(0)
\end{aligned}
$$

where $(\mathrm{x}, \lambda)$ - solution problem Cauchy (1.3) or (1.5) and $\mathrm{x}_{\mathrm{k}, \lambda}$ - the zeros of the solutions.

\section{Main result}

Unless otherwise stated, suppose that for each $\lambda>$ $1, \mathrm{n}:=[\sqrt{\lambda}], \Omega:=\sqrt{\lambda}$ and $\mathrm{x}_{\mathrm{k}, \lambda}:=\mathrm{k} \pi / \sqrt{\lambda}$ and $\mathrm{l}_{\mathrm{k}, \lambda}(\mathrm{x}):=\frac{(-1)^{\mathrm{k}} \sin \Omega \mathrm{x}}{\Omega \mathrm{x}-\mathrm{k} \pi}$. Let $\Omega$ set of real continuous non decreasing convex up on $[0, b-a]$, vanishing at zero functions $\omega$. Let $C\left(\omega^{1},[a, b]\right)$ and $C\left(\omega^{r},[a, b]\right)$ is the set of elements of $C[a, b]$ such that for any $x$ and $\mathrm{x}+\mathrm{h}(\mathrm{a} \leq \mathrm{x}<\mathrm{x}+\mathrm{h} \leq \mathrm{b})$ we have the equalities

$$
\begin{gathered}
f(x+h)-f(x) \geq-K_{f} \omega(h) \\
\text { or } f(x+h)-f(x) \leq K_{f} \omega(h),
\end{gathered}
$$

accordingly. Where $\omega \in \Omega$. Selecting positive constants $\mathrm{K}_{\mathrm{f}}$ may depend only on the function $\mathrm{f}$. In this case the function $\omega(\mathrm{h})$ is sometimes referred to, accordingly, the left-hand or right-hand continuity module. In principle, the definition of a unilateral module of continuity could be considered any functions $\omega(\mathrm{h})$ vanishing at zero, continuous on $[0, b-a]$ or $[0, \infty)$. The wording of all the results of this work in this case, would remain in force. Without loss of generality, in the definition of unilateral modulus of continuity (2.1) can be considered $\omega \in \Omega$.

Classic modulus of continuity $f \in C[a, b]$ denoted as usual $\omega(\mathrm{f}, \delta)=\sup _{|\mathrm{h}|<\delta: x, x+h \in[a, b]}|f(x+h)-f(x)|$.

The module of continuity of $\mathrm{f} \in \mathrm{C}[0, \pi]$, if $\mathrm{a}=0, \mathrm{~b}=\pi$ will denote 
$\omega_{1} \quad(\mathrm{f}, \quad \delta)=\sup _{|\mathrm{h}|<\delta: \mathrm{x}, \mathrm{x}+\mathrm{h} \in[0, \pi]} \mid \mathrm{f}(\mathrm{x}+\mathrm{h})-$ $f(x) \mid$.Module of change of $f$ on the interval $[a, b]$ is called function defined by the equation

$$
v(n, f)=\sup _{T_{n}} \sum_{k=0}^{n-1}\left|f\left(t_{k+1}\right)-f\left(t_{k}\right)\right|
$$

where $\quad \mathrm{T}_{\mathrm{n}}=\left\{\mathrm{a} \leq \mathrm{t}_{0}<\mathrm{t}_{1}<\mathrm{t}_{2}<\cdots<\mathrm{t}_{\mathrm{n}-1}<\right.$ $\left.\mathrm{t}_{\mathrm{n}} \leq \mathrm{b}\right\}, \mathrm{n} \in \mathrm{N}$.

Take a non-negative, non-decreasing convex up function of a natural argument to $v(n)$. If a module of changes of function $f$ on the interval $[a, b]$, such that $\mathrm{v}(\mathrm{n}, \mathrm{f})=\mathrm{O}(\mathrm{v}(\mathrm{n}))$ with $\mathrm{n} \rightarrow \infty$, then we say that $\mathrm{f}$ belongs to the class $V(v)$. Here, also, the choice of uniformity of the constants o-symbolism can only depend on $f$.

By analogy with the positive (negative) change of function will be called positive (negative) module of change of function $f$ on the interval $[a, b]$, accordingly, the function of a natural argument type

$$
\begin{aligned}
\mathrm{v}^{+}(\mathrm{n}, \mathrm{f}) & =\sup _{\mathrm{T}_{\mathrm{n}}} \sum_{\mathrm{k}=0}^{\mathrm{n}-1}\left(\mathrm{f}\left(\mathrm{t}_{\mathrm{k}+1}\right)-\mathrm{f}\left(\mathrm{t}_{\mathrm{k}}\right)\right)_{+} \\
\text {and } \mathrm{v}^{-}(\mathrm{n}, \mathrm{f}) & =\inf _{\mathrm{T}_{\mathrm{n}}} \sum_{\mathrm{k}=0}^{\mathrm{n}-1}\left(\mathrm{f}\left(\mathrm{t}_{\mathrm{k}+1}\right)-\mathrm{f}\left(\mathrm{t}_{\mathrm{k}}\right)\right)_{-},
\end{aligned}
$$$$
\text { where } \quad \mathrm{z}_{+}=\frac{\mathrm{z}+|\mathrm{z}|}{2} \text { and } \mathrm{z}_{-}=\frac{\mathrm{z}-|\mathrm{z}|}{2}
$$$$
\text { and } \quad \mathrm{T}_{\mathrm{n}}=\left\{\mathrm{a} \leq \mathrm{t}_{0}<\mathrm{t}_{1}<\mathrm{t}_{2}<\cdots<\mathrm{t}_{\mathrm{n}-1}<\right.
$$
$\left.t_{n} \leq b\right\}, n \in N$. We say that $f$ belongs to the class of $\mathrm{V}^{+}(\mathrm{v})$ or $\mathrm{V}^{-}(\mathrm{v})$, if there exists a constant $\mathrm{M}_{\mathrm{f}}$, that for any natural $\mathrm{n}$ true inequality

$$
\mathrm{v}^{+}(\mathrm{n}, \mathrm{f}) \leq \mathrm{M}_{\mathrm{f}} \mathrm{v}(\mathrm{n}) \quad \text { or } \quad \mathrm{v}^{-}(\mathrm{n}, \mathrm{f}) \geq-\mathrm{M}_{\mathrm{f}} \mathrm{v}(\mathrm{n})
$$

accordingly.

We define two functional classes. The function $\mathrm{f}$ $\in \mathrm{C}\left(\omega^{\mathrm{l}} \quad[\mathrm{a}, \mathrm{b}]\right) \cap \mathrm{V}^{-}(\mathrm{v}) \quad\left(\mathrm{f} \in \mathrm{C}\left(\omega^{\mathrm{r}}[\mathrm{a}, \mathrm{b}]\right) \cap \mathrm{V}^{+}(\mathrm{v})\right)$ here, $0<\mathrm{a}<\mathrm{b}<\pi, \quad 0<\varepsilon<(\mathrm{b}-\mathrm{a}) / 2$, if there are $\mathrm{a}$ nondecreasing concave function of a natural argument $v$ (n) and the function $\omega \in \Omega$ such that

$$
\begin{gathered}
\lim _{\mathrm{n} \rightarrow \infty} \min _{1 \leq \mathrm{m} \leq \mathrm{k}_{2}-\mathrm{k}_{1}-1}\left\{\omega\left(\frac{\pi}{\sqrt{\lambda}}\right) \sum_{\mathrm{k}=1}^{\mathrm{m}} \frac{1}{\mathrm{k}}+\sum_{\mathrm{k}=\mathrm{m}+1}^{\mathrm{k}_{2}-\mathrm{k}_{1}-1} \frac{\mathrm{v}(\mathrm{k})}{\mathrm{k}^{2}}\right\} \\
=0,
\end{gathered}
$$

where $\mathrm{k} 1, \mathrm{k} 2+1$ - the smallest and largest number of nodes $\mathrm{x}_{\mathrm{k}, \lambda}=\mathrm{k} \pi / \Omega$, falling in the interval [a,b], and

$\left.\left.\mathrm{f} \in \mathrm{C}\left(\omega^{\mathrm{l}},[\mathrm{a}, \mathrm{b}]\right)\right]\right) \cap \mathrm{V}^{-}(\mathrm{v})\left(\mathrm{f} \in \mathrm{C}\left(\omega^{\mathrm{r}}[\mathrm{a}, \mathrm{b}]\right) \cap\right.$ $\left.\mathrm{V}^{+}(\mathrm{v})\right)$.

The description of the first class contains a restriction only on decreasing the function. The description of the second class contains a restriction only on the increase of the function.

Theorem 2.1. If $\left.\left.f \in C\left(\omega^{l}[a, b]\right)\right]\right) \cap V^{-}(v)$ (f $\left.\in \mathrm{C}\left(\omega^{r}[\mathrm{a}, \mathrm{b}]\right) \cap V^{+}(v)\right)$, here, $0<\mathrm{a}<\mathrm{b}<\mathrm{n}, 0<\varepsilon<$ $(\mathrm{b}-\mathrm{a}) / 2$, then

$$
\begin{gathered}
\lim _{\Omega \rightarrow \infty}\left\|f-C_{\Omega}(f, .)\right\|_{C[a+\varepsilon, b-\varepsilon]} \\
=0 .
\end{gathered}
$$

Where operator $C_{\Omega}(f,$.$) defined in (1.1).$

Remark 2.2. On the set $[0, \pi] \backslash[\mathrm{a}, \mathrm{b}]$ ratio (1.1) can be not performed (See ${ }^{[22]}$ ).

We present auxiliary results, which will be used in the future.

Proposition 2.3 ${ }^{[23]}$, Proposition 9). Let $y(x, \lambda)$ be the solution of Cauchy problem (1.5) or (1.6) and assume that in case of the Cachy problem (1.5) relations (1.2) hold, while in the case of (1.6) relations (1.4) hold. If $f \in C_{0}[0, \pi]$, then

$$
\begin{aligned}
& \lim _{\lambda \rightarrow \infty}\left(f(x)-S_{\lambda}(f, x)-\frac{1}{2} \sum_{k=0}^{n-1}\left(f\left(x_{k+1, \lambda}\right)\right.\right. \\
& \left.\left.\quad-f\left(x_{k, \lambda}\right)\right) S_{k \cdot \lambda}(x)\right) \\
& \quad=0,
\end{aligned}
$$

For any $0 \leq a<b \leq \pi, 0<\varepsilon<(b-a) / 2$ denoted

$Q_{\lambda}(f,[a, b], \varepsilon)$ :

$=\max _{p_{1} \leq p \leq p_{2}}\left|\sum_{m=m_{1}}^{m_{2}} \frac{\mathfrak{f}\left(x_{2 m+1, \lambda}\right)-f\left(x_{2 m, \lambda}\right)}{\mathrm{p}-2 m}\right|$.

Here the dashes on the summation signs in (2.5) mean that are no terms with zero denominator. Where $\mathrm{p}_{1}$, $\mathrm{p}_{2}, \mathrm{~m}_{1}$ and $\mathrm{m}_{2}$ are the indices of the zeros determined by the inequalities

$$
\begin{array}{cr}
\mathrm{x}_{\mathrm{p} 1, \lambda} \leq \mathrm{a}+\varepsilon<\mathrm{x}_{\mathrm{p} 1+1, \lambda}, & \mathrm{x}_{\mathrm{p} 2, \lambda} \leq \mathrm{b}-\varepsilon<\mathrm{x}_{\mathrm{p} 2+1, \lambda}, \\
\mathrm{x}_{\mathrm{k} 1-1, \lambda}<\mathrm{a} \leq \mathrm{x}_{\mathrm{k} 1, \lambda}, & \mathrm{x}_{\mathrm{k} 2+1, \lambda} \leq \mathrm{b}<\mathrm{x}_{\mathrm{k} 2+2, \lambda}, \\
m_{1}=\left[\frac{k_{1}}{2}\right]+1, & m_{2}=\left[\frac{k_{2}}{2}\right] .
\end{array}
$$

Here $[z]$ denote the integer part z.

Proposition 2.4. If $f$ function $f \in C[0, n]$, then from a ratio

$$
\begin{gathered}
\lim _{\lambda \rightarrow \infty} Q_{\lambda}(f,[a, b], \varepsilon) \\
=0
\end{gathered}
$$

follows (2.3).

Proof of Proposition 2.4. We denote

$$
\begin{aligned}
& \psi_{k, \lambda}=f\left(x_{k+1}, \lambda\right)-f\left(x_{k}, \lambda\right) \\
& k_{1} \leq k \leq k_{2} ; \lambda>0 .
\end{aligned}
$$

We take into account that we have the estimate $\left|\psi_{k, \lambda}\right|=\left|f\left(x_{k+1}, \lambda\right)-f\left(x_{k}, \lambda\right)\right| \leq \omega\left(f, \frac{\pi}{\sqrt{\lambda}}\right)$ for all $\mathrm{k}_{1} \leq \mathrm{k} \leq \mathrm{k}_{2} ; \lambda>0$.

We fix an arbitrary $x \in[a+\varepsilon, b-\varepsilon]$. Choose in- 
$\operatorname{dex} \mathrm{p}=\mathrm{p}(\mathrm{x}, \lambda)$, so that $\mathrm{x} \in\left[x_{p \lambda,}, x_{p+1, \lambda}\right)$. Then $x=$ $x_{p \lambda,}+\frac{a \pi}{\sqrt{\lambda}}$, where $\mathrm{a}=\mathrm{a}(\mathrm{x}, \lambda) \in[0,1)$

$$
x-x_{k, \lambda}=\frac{p-k+a}{\sqrt{ } \lambda} \pi \text {. }
$$

From (2.8) for all $x \in[a+\varepsilon, b-\varepsilon]$ we have the estimate

$$
\begin{aligned}
& \left|\sum_{\substack{k: k_{1} \leq k \leq k_{2} ; \\
|p-k| \geq 3 ;}} \frac{(-1)^{k} \psi_{k, \lambda}}{p-k+a}-\sum_{\substack{k: k_{1} \leq k \leq k_{2} ; \\
|p-k| \geq 3 ;}} \frac{(-1)^{k} \psi_{k, \lambda}}{p-k}\right| \leq \\
& \quad \omega\left(f, \frac{\pi}{\sqrt{\lambda}}\right) \sum_{\substack{k: k_{1} \leq k \leq k_{2} ; \\
|p-k| \geq 3 ;}} \frac{a}{|p-k|(|p-k|-1)} \\
& \leq \omega\left(f, \frac{\pi}{\sqrt{\lambda}}\right) .
\end{aligned}
$$

Notice, that if $\mathrm{h}(\lambda)=\sqrt{\lambda}, \mathrm{q}_{\lambda} \equiv 0$ solution of the Cauchy problem (1.5) is $\mathrm{y}(\mathrm{x}, \lambda)=\sin \sqrt{\lambda x}$.

We take into account (2.7). We decompose the sum in (2.4) as follows:

$$
\begin{aligned}
& \frac{1}{2} \sum_{k=k_{1}}^{k_{2}}\left(f\left(x_{k+1, \lambda}\right)-f\left(x_{k, \lambda}\right)\right) l_{k, \lambda} \\
& +\frac{1}{2} \sum_{\substack{k \in[0, \lambda-1] \backslash\left[k_{1}, k_{2}\right] \\
\frac{1}{2}}}\left(f\left(x_{k+1, \lambda}\right)-f\left(x_{k, \lambda}\right)\right) l_{k, \lambda}(x)= \\
& +\frac{1}{2} \sum_{k \in k_{1} \leq k \leq k_{2} ;} \psi_{k, \lambda}, l_{k, \lambda}(x)+\frac{1}{2} \sum_{\substack{k: k_{1} \leq k \leq k_{2} ; \\
|p-k|<3 ;}} \psi_{k, \lambda}, l_{k, \lambda}(x) \\
& \sum_{k \in[0, \lambda-1] \backslash\left[k_{1}, k_{2}\right]} \psi_{k, \lambda}, l_{k, \lambda}(x) .
\end{aligned}
$$

Now, using the triangle inequality, of (2.7), (2.9) uniformly for $x \in[\mathrm{a}+\varepsilon, \mathrm{b}-\varepsilon]$ the estimate

$$
\begin{gathered}
\mid \frac{1}{2} \sum_{k=k_{1}}^{k_{2}}\left(f\left(x_{k+1, \lambda}\right)-f\left(x_{k, \lambda}\right)\right) l_{k, \lambda} \\
-\frac{\sin \sqrt{\lambda x}}{2 \pi} \sum_{k=k_{1}}^{k_{2}} \frac{(-1)^{k} \psi_{k, \lambda} \mid}{p-k} \mid \leq \\
\frac{1}{2 \pi}\left|\sum_{k:|p-k| \geq 3} \frac{(-1)^{k} \psi_{k, \lambda}}{p-k+a}-\sum_{k:|p-k| \geq 3} \frac{(-1)^{k} \psi_{k, \lambda}}{p-k}\right|+ \\
\sum_{k:|p-k|<3}\left|\psi_{k, \lambda} l_{k, \lambda}(x)\right|+\frac{1}{2 \pi} \sum_{k:|p-k|<3} \frac{|| \psi_{k, \lambda} \mid}{|p-k|} \\
\leq \frac{5}{\pi} \omega\left(f, \frac{\pi}{\sqrt{\lambda}}\right) .
\end{gathered}
$$

There are a constant $\mathrm{C}$ and number $\mathrm{n}_{0} \in \mathrm{N}$ independent of function $f \in \mathrm{C}[0, \pi], 0 \leq \mathrm{a}<\mathrm{b} \leq \pi$ and $0<\varepsilon$ $<(\mathrm{b}-\mathrm{a}) / 2$, such that for all $\mathrm{x} \in[\mathrm{a}+\varepsilon, \mathrm{b}-\varepsilon]$ and $\mathrm{n}>\mathrm{n}_{0}$ the inequality is fair

$$
\begin{gathered}
\left|\frac{1}{2} \sum_{k \in[0, n-1] \backslash\left[k_{1}, k_{2}\right]} \psi_{k, \lambda} l_{k, \lambda}(x)\right| \\
\leq \frac{\omega_{1}\left(f, \frac{\pi}{\sqrt{\lambda}}\right)}{2} \sum_{k \in[0, n-1] \backslash\left[k_{1}, k_{2}\right]}\left|l_{k, \lambda}(x)\right| \leq \\
C_{\omega_{1}}\left(f, \frac{\pi}{\sqrt{\lambda}}\right) \ln \frac{2 \pi}{\varepsilon} .
\end{gathered}
$$

Thence, by (2.11) (2.4) we have for all $\mathrm{x} x \in[\mathrm{a}+$ $\varepsilon, \mathrm{b}-\varepsilon]$ ratio

$$
\begin{gathered}
\lim _{n \rightarrow \infty}\left(f(x)-C_{\Omega}(f, x)-\frac{\sin \sqrt{\lambda x}}{2 \pi} \sum_{k=k_{1}}^{k_{2}} \frac{(-1)^{k} \psi_{k, \lambda}}{p-k}\right) \\
=0 .
\end{gathered}
$$

We estimate the last term in (2.12) by means of ratio (2.8) and triangle inequality

$$
\begin{gathered}
\left|\frac{\sin \sqrt{\lambda x}}{2 \pi} \sum_{k=k_{1}}^{k_{2}} \frac{(-1)^{k} \psi_{k, \lambda}}{p-k}\right| \\
\leq 2\left|\frac{1}{2 \pi} \sum_{m=m_{1}}^{m_{2}} \frac{\psi_{2 m, \lambda}}{p-2 m}\right|+\left|\frac{1}{2 \pi} \sum_{k=k_{1}}^{k_{2}} \frac{\psi_{k, \lambda}}{p-k}\right| \\
+O\left(\omega\left(f, \frac{1}{\sqrt{\lambda}}\right)\right) .
\end{gathered}
$$

By the continuity of $f$ there exists a sequence of positive integers $\left\{l_{n}\right\}_{n=1}^{\infty}$, such that

$$
\begin{gathered}
l_{n}=o(n), \quad \lim _{n \rightarrow \infty} l_{n}=\infty, \quad \lim _{\lambda \rightarrow \infty} \omega\left(f, \frac{1}{\sqrt{\lambda}}\right) \sum_{k=1}^{l_{n}} \frac{1}{k} \\
=0, \quad n:=[\lambda] .
\end{gathered}
$$

We estimate the second sum in (2.13)

$$
\begin{aligned}
\left|\frac{1}{2 \pi} \sum_{k=k_{1}}^{k_{2}} \frac{\psi_{k, \lambda}}{p-k}\right| & \leq\left|\frac{1}{2 \pi} \sum_{k:|p-k| \leq l_{n}} \frac{\psi_{k, \lambda}}{p-k}\right| \\
& +\left|\frac{1}{2 \pi} \sum_{k:|p-k|>l_{n}} \frac{\psi_{k, \lambda}}{p-k}\right| .
\end{aligned}
$$

From here and inequalities (2.8) follows

$$
\begin{aligned}
\left|\frac{1}{2 \pi} \sum_{k:|p-k| \leq l_{n}} \frac{\psi_{k, \lambda}}{p-k}\right| \leq \frac{1}{2 \pi} \sum_{k:|p-k| \leq l_{n}}\left|\frac{\psi_{k, \lambda}}{p-k}\right| \\
\leq \frac{1}{\pi} \omega\left(f, \frac{\pi}{\sqrt{\lambda}}\right) \sum_{k=1}^{l_{n}} \frac{1}{k} .
\end{aligned}
$$

Hence by (2.15) after taking the Abel transform in case $\mathrm{k} \in\left[\mathrm{k}_{1}, \mathrm{k}_{2}\right]:|\mathrm{p}-\mathrm{k}|>1_{\mathrm{n}}$ we obtain the estimate 


$$
\begin{aligned}
\left|\frac{1}{2 \pi} \sum_{k:|p-k|>1_{n}} \frac{\psi_{k, \lambda}}{p-k}\right| & \frac{4\|f\|_{C[a, b]}}{l_{n}+1} \\
& +4\|f\|_{C[a, b]} \sum_{k=l_{n}}^{\infty} \frac{1}{k(k+1)} .
\end{aligned}
$$

Hence by (2.14), (2.15) and (2.16) we obtain the uniform estimate for all $\mathrm{x} \in[\mathrm{a}+\varepsilon, \mathrm{b}-\varepsilon]$

$$
\left|\frac{1}{2 \pi} \sum_{k=k_{1}}^{k_{2}} \frac{\psi_{k, \lambda}}{p-k}\right|=o(1) \text {. }
$$

Notice, that if $h(\lambda)=\sqrt{\lambda}, q_{\lambda} \equiv 0$ solution of the Cauchy problem (1.5) is $y(x, \lambda)=\sin \sqrt{\lambda} x$, Then by (2.4), (2.5), (2.12), (2.13), (2.17) and triangle inequality we obtain the relation

$$
\begin{gathered}
\left|f(x)-C_{\Omega}(f, x)\right| \\
\leq\left|f(x)-C_{\Omega}(f, x)-\frac{\sin \sqrt{\lambda x}}{2 \pi} \sum_{k=k_{1}}^{k_{2}} \frac{(-1)^{k} \psi_{k, \lambda}}{p-k}\right|+ \\
\left|\frac{1}{\pi} \sum_{m=m_{1}}^{m_{2}} \frac{\psi_{2 m, \lambda}}{p-2 m}\right|_{\leq}+\left|\frac{1}{2 \pi} \sum_{k=k_{1}}^{k_{2}} \frac{\psi_{2 m, \lambda}}{p-2 m}\right|+O\left(\omega\left(f, \frac{1}{\sqrt{\lambda}}\right)\right) \\
\frac{1}{\pi} Q_{\lambda}(f,[a, b], \varepsilon)+o(1) .
\end{gathered}
$$

From which it follows the sufficiency (2.6) for uniform convergence (2.3). Proposition 2.4 proved.

For all $0 \leq \mathrm{a}<\mathrm{b} \leq \pi, 0<\varepsilon<(\mathrm{b}-\mathrm{a}) / 2$ denoted $Q_{\lambda}^{*}(f,[a, b], \varepsilon)$ :

$=\max _{p_{1} \leq p \leq p_{2}} \sum_{m=m_{1}}^{m_{2}}\left|\frac{f\left(x_{2 m+1, \lambda}\right)-f\left(x_{2 m, \lambda}\right)}{p-2 m}\right|$.

Proposition 2.5. If function $f \in C[0, \pi]]$, then the ratio of

$$
\lim _{n \rightarrow \infty} Q_{\lambda}^{*}(f,[a, b], \varepsilon)=0
$$

implies (2.3).

Proof. Indeed, by Proposition 2.4 satisfy the condition (2.19) implies truth of the saying (2.6) and therefore, the ratio (2.3).

Remark 2.6. Propositions 2.4 and 2.5 are analogues of known signs of A.A. Pri-valov uniform convergence of trigonometric polynomial and algebraic interpolations polynomial Lagrange with the matrix of interpolation nodes P.L. Chebyshev ${ }^{[33]}$.

Proof of the Theorem 2.1 Let the function $\mathrm{v}$ and $\mathrm{w}$ satisfies the condition (2.2) and $\mathrm{f} \in \mathrm{C}\left(\omega^{l} 1[\mathrm{a}, \mathrm{b}]\right) \cap$ $V^{-}(\mathrm{v})$.We show that the relation (2.19) is true. By virtue of the uniform continuity and boundedness of $\mathrm{f}$, for any positive $\epsilon$ there exist natural numbers $\mathrm{v} n_{1}$ such that for all $\lambda \geq n_{1}(\lambda \in \mathrm{R})$ simultaneously take place two inequalities

$$
\omega\left(f, \frac{\pi}{\sqrt{\lambda}}\right) \sum_{k=1}^{v} \frac{1}{k}<\frac{\epsilon}{6}
$$

and

$$
24\|f\|_{C[a, b]}<\epsilon v .
$$

Let $\lambda \geq \mathrm{n}_{1}$. We find $\mathrm{p}_{\mathrm{o}}$, depending on $\mathrm{n}, \mathrm{a}, \mathrm{b}, \varepsilon$ and $\mathrm{f}$ at which the maximum in the definition (2.18)

$$
Q_{\lambda}^{*}(f,[a, b], \varepsilon)=\sum_{m=m_{1}}^{m_{2}}\left|\frac{f\left(x_{2 m+1, \lambda}\right)-f\left(x_{2 m, \lambda}\right)}{p_{0}-2 m}\right| .
$$

Assuming that

$$
Q_{\lambda}^{* *}(f,[a, b], \varepsilon):=\sum_{k=k_{1}}^{k_{2}}\left|\frac{f\left(x_{k+1, \lambda}\right)-f\left(x_{k, \lambda}\right)}{p_{0}-k}\right| .
$$

The value of $Q_{\lambda}^{* *}(f,[a, b], \varepsilon)$ is obtained from $Q_{\lambda}^{*}(f,[a, b], \varepsilon)$ by the addition of non-negative terms, therefore is fair the inequality

$$
Q_{\lambda}^{*}(f,[a, b], \varepsilon) \leq Q_{\lambda}^{* *}(f,[a, b], \varepsilon) .
$$

We divide $Q_{\lambda}^{* *}(f,[a, b], \varepsilon)$ into two terms

$$
\begin{gathered}
Q_{\lambda}^{* *}(f,[a, b], \varepsilon):=\sum_{k=k_{1}}^{k_{2}} \backslash\left|\frac{f\left(x_{k+1, \lambda}\right)-f\left(x_{k, \lambda}\right)}{p_{0}-k}\right|- \\
2 \sum_{k=k_{1}}^{k_{2}} \cdots \frac{f\left(x_{k+1, \lambda}\right)-f\left(x_{k, \lambda}\right)}{p_{0}-k}=S_{1}\left(p_{0}\right)+S_{2}\left(p_{0}\right),
\end{gathered}
$$

where two strokes mean that in the sum are absent non-negative summands and with index $\mathrm{k}=\mathrm{p}_{0}$.

First, we estimate the first sum. Representing it in the form

$$
\begin{gathered}
S_{1}\left(p_{0}\right) \sum_{\substack{k: k \in\left[k_{1}, k_{2}\right], 0<\left|p_{0}-k\right|<v}} \frac{f\left(x_{k+1, \lambda}\right)-f\left(x_{k, \lambda}\right)}{p_{0}-k}+ \\
\sum_{\substack{k: k \in\left[k_{1}, k_{2}\right], 0<\left|p_{0}-k\right| \geq v}} \frac{f\left(x_{k+1, \lambda}\right)-f\left(x_{k, \lambda}\right)}{p_{0}-k} \\
=S_{1,1(p o)}+S_{1,2(p o) .}
\end{gathered}
$$

In the case $\left\{\mathrm{k}: \mathrm{k} \in\left[k_{1}, k_{2}\right], 0<\left|\mathrm{p}_{0}-\mathrm{k}\right| \geq v\right\}=$ $\emptyset$ believe that the second term is zero.

From the inequality (2.20) have

$$
\left|S_{1,1}\left(p_{0}\right)\right| \leq 2 \omega\left(f, \frac{\pi}{\sqrt{\lambda}}\right) \sum_{k=1}^{v} \frac{1}{k}<\frac{\epsilon}{3} \text {. }
$$

We now estimate the amount $S_{1,2}\left(p_{0}\right)$. If $p_{0}$ such that inequalities are fair $\mathrm{k}_{1} \leq \mathrm{p}_{0}-\mathrm{v}<\mathrm{p}_{0}<\mathrm{p}_{0}+\mathrm{v} \leq \mathrm{k}_{2}$, 
then ratios take place $\mathrm{p}_{0}-\mathrm{k}_{1} \geq v \mathrm{k}_{2}-\mathrm{p}_{0} \geq v$. Hence by (2.21), after taking the Abel transform we obtain estimate

$$
\begin{gathered}
\left|S_{1,2}\left(p_{0}\right)\right| \leq\left|\sum_{k=k_{1}}^{p_{0}-v} \frac{f\left(x_{k+1, \lambda}\right)-f\left(x_{k, \lambda}\right)}{p_{0}-k}\right| \\
+\left|\sum_{k=p_{0}+v}^{k_{2}} \frac{f\left(x_{k+1, \lambda}\right)-f\left(x_{k, \lambda}\right)}{k-p_{0}}\right| \\
\mid \begin{array}{c}
\sum_{k=k_{1}}^{p_{0}-v-1} \frac{f\left(x_{k+1, \lambda}\right)-f\left(x_{k, \lambda}\right)}{\left(p_{0}-k\right)\left(p_{0}-k-1\right)} \mid \\
+\sum_{k=p_{0}+v} \frac{f\left(x_{k+1, \lambda}\right)-f\left(p_{0}+v_{, \lambda}\right)}{\left(k-p_{0}\right)(k+1, \lambda)-f\left(x_{k_{1}, \lambda}\right)} \mid+ \\
\left.k_{0}-p_{0}\right) \mid \\
4\|f\|_{C[a, b]} \sum_{i=v}^{\infty} \frac{1}{i(i+1)}+\frac{4\left(x_{k_{2}, \lambda}\right)-f\left(x_{p_{0}+v, \lambda}\right)}{k_{2}-p_{0}} \mid \leq \\
<\frac{\epsilon\|f\|_{C[a, b]}}{v} \leq \frac{8\|f\|_{C[a, b]}}{v}
\end{array}
\end{gathered}
$$

Similarly we prove (2.26), if p0 would be so, that will be inequality $p_{0}-v<\mathrm{k}_{1} \leq p_{0}<p_{0}+v \leq \mathrm{k}_{2}$ or inequality $\mathrm{k}_{1} \leq p_{0}-v<p_{0} \leq \mathrm{k}_{2}<p_{0}+v$. Of the possible variant remained only when $p_{0^{-}} \mathrm{v}<\mathrm{k}_{1}<p_{0}<\mathrm{k}_{2}<p_{0}$ $+\mathrm{v}$. In this situation, we have $\left|\mathrm{S}_{1,2}\left(p_{0}\right)\right|=0$.

From (2.24), (2.25) end (2.26) we obtain inequality

$$
\left|S_{1}\left(p_{0}\right)\right| \leq \frac{2^{\sim} \epsilon}{3}
$$

for all $\lambda \geq \mathrm{n}_{1}$.

Let's move on to the study of the properties of the sum $\mathrm{S}_{2}\left(\mathrm{p}_{0}\right)$. Take any integer $\mathrm{m}: 1 \leq \mathrm{m} \leq \mathrm{k}_{2}-\mathrm{k}_{1}-2$ and represented $S_{2}\left(p_{0}\right)$ in the form

$$
\begin{aligned}
& 0 \leq S_{2}\left(p_{0}\right)=-2 \\
& \sum_{\substack{k: k \in\left[k_{1}, k_{2}\right],\left|p_{0}-k\right| \leq m}} \frac{\cdots f\left(x_{k+1, \lambda}\right)-f\left(x_{k, \lambda}\right)}{\left|p_{0}-k\right|}- \\
& 2 \sum_{\substack{k: k \in\left[k_{1}, k_{2}\right],\left|p_{0}-k\right|>m}} \frac{\cdots f\left(x_{k+1, \lambda}\right)-f\left(x_{k, \lambda}\right)}{\left|p_{0}-k\right|} \\
& =S_{2,1}\left(p_{0}\right)+S_{2,2}\left(p_{0}\right) \text {. }
\end{aligned}
$$

Function $\mathrm{f} \in \mathrm{C}\left(\omega^{l}[\mathrm{a}, \mathrm{b}]\right)$, therefore by definition (2.1) we have relation

\section{Therefore}

$$
f\left(x_{k+1, \lambda}\right)-f\left(x_{k, \lambda}\right) \geq-K_{f} \omega\left(\frac{\pi}{\sqrt{\lambda}}\right) .
$$

$$
0 \leq S_{2,1}\left(p_{0}\right)=-2
$$

$$
\begin{aligned}
& \sum_{\substack{k: k \in\left[k_{1}, k_{2}\right],\left|p_{0}-k\right| \leq m}} \frac{\cdots f\left(x_{k+1, \lambda}\right)-f\left(x_{k, \lambda}\right)}{\left|p_{0}-k\right|} \leq \\
& \quad 4 K_{f \omega}\left(\frac{\pi}{\sqrt{\lambda}}\right) \sum_{k=1}^{m} \frac{1}{k} .
\end{aligned}
$$

We estimate the amount $S_{2,2}\left(p_{0}\right)$.

$$
\sum_{\substack{k: k \in\left[k_{1}, k_{2}\right],\left|p_{0}-k\right|>m}} \frac{\dddot{ } \leq S_{2,2}\left(p_{0}\right)=-2}{\left.\mid p_{k+1, \lambda}\right)-f\left(x_{k, \lambda}\right)} \leq
$$$$
2 \sum_{k=k_{1}}^{p_{0}-m-1} \frac{-\left(f\left(x_{k+1, \lambda}\right)-f\left(x_{k, \lambda}\right)\right)_{-}}{p_{0}-k}
$$$$
+2 \sum_{k=p_{0}+m+1}^{k_{2}} \frac{-\left(f\left(x_{k+1, \lambda}\right)-f\left(x_{k, \lambda}\right)\right)_{-}}{k-p_{0}} \text {. }
$$

Note that $p_{0}-\mathrm{m}<\mathrm{k}_{1}$ or $\mathrm{p}_{0}+\mathrm{m}>\mathrm{k}_{2}$, then in (2.30) disappears respectively, the first or second term. In case that $p_{0}-\mathrm{m}<\mathrm{k}_{1}<\mathrm{k}_{2}<\mathrm{p}_{0}+\mathrm{m}$, sum $\mathrm{S}_{2,2}\left(\mathrm{p}_{0}\right)$ in (2.28) absent. Take into account that $\mathrm{f} \in \mathrm{V}$ (v). We will apply Abel's transformation in estimate (2.30)

$$
\begin{aligned}
& 0 \leq S_{2,2}\left(p_{0}\right) \leq \\
& 2\left(\frac{\sum_{k=k_{1}}^{p_{0}-m-1}-\left(f\left(x_{k+1, \lambda}\right)-f\left(x_{k, \lambda}\right)\right)_{-}}{p_{0}-k_{1}}\right. \\
& +\sum_{k=k_{1}+1}^{p_{0}-m-1} \frac{\sum_{j=k}^{p_{0}-m-1}-\left(f\left(x_{k+1, \lambda}\right)-f\left(x_{k, \lambda}\right)\right)_{-}}{\left(p_{0}-k\right)\left(p_{0}-k+1\right)} \\
& +\frac{\sum_{k=p_{0}+m+1}^{p_{0}-m-1}-\left(f\left(x_{k+1, \lambda}\right)-f\left(x_{k, \lambda}\right)\right)_{-}}{k_{2}-p_{0}} \\
& \left.+\sum_{k=p_{0}+m}^{k_{2}-1} \frac{\sum_{j=p_{0}+m+1}^{k}-\left(f\left(x_{k+1, \lambda}\right)-f\left(x_{k, \lambda}\right)\right)_{-}}{\left(p_{0}-k\right)\left(p_{0}-k-1\right)}\right) \leq \\
& 2\left(\frac{\left(\left(p_{0}-k_{1}\right)-m-1\right) K_{f \omega}\left(\frac{\pi}{\sqrt{\lambda}}\right)}{\left(p_{0}-k_{1}\right)}\right. \\
& \left.+M_{f} \sum_{k=p_{0}+m+1}^{k_{2}-1} \frac{v\left(k-p_{0}-m\right)}{\left(p_{0}-k\right)\left(p_{0}-k-1\right)}\right) \leq \\
& 2 M_{f}\left(\sum_{k=m+1}^{p_{0}-k_{1}-1} \frac{v(k-m)}{k(k+1)}+\sum_{k=m+1}^{k_{2}-p_{0}-1} \frac{v(k-m)}{k(k+1)}\right) \\
& +4 K_{f \omega}\left(\frac{\pi}{\sqrt{\lambda}}\right) \\
& \leq 4 M_{f} \sum_{k=m+1}^{k_{2}-k_{1}-1} \frac{v(k)}{k^{2}}+4 K_{f \omega}\left(\frac{\pi}{\sqrt{\lambda}}\right) \text {. }
\end{aligned}
$$

Hence (2.28), (2.29) and (2.30) we have 


$$
\begin{aligned}
0 \leq S_{2}\left(p_{0}\right) \leq 4 & K_{f} \omega\left(\frac{\pi}{\sqrt{\lambda}}\right) \sum_{k=1}^{m} \frac{1}{k} \\
& +4 M_{f} \sum_{k=m+1}^{k_{2}-k_{1}-1} \frac{v(k)}{k^{2}}+4 K_{f \omega}\left(\frac{\pi}{\sqrt{\lambda}}\right) .
\end{aligned}
$$

Conditions (2.2), due to the non-negativity of both summands, equivalent to

$\lim _{n \rightarrow \infty} \min _{1 \leq m \leq k 2-k 1-1} \max \left\{\omega\left(\frac{\pi}{\sqrt{\lambda}}\right) \sum_{k=1}^{m} \frac{1}{k}, \sum_{k=m+1}^{k_{2}-k_{1}-1} \frac{v(k)}{k^{2}}\right\}$ $=0$.

Therefore exists an $\mathrm{n}_{2} \in \mathrm{N}, \mathrm{n}_{2} \geq \mathrm{n}_{1}$, that for avery $\lambda \geq \mathrm{n}_{2}$ there are $\mathrm{m}: 1 \leq \mathrm{m} \leq \mathrm{k}_{2}-\mathrm{k}_{1}-1$ for which the inequality

$$
0 \leq S_{2}\left(p_{0}\right) \leq \frac{\epsilon}{3}
$$

As result of by (2.22), (2.23), (2.24), (2.27) and (2.31) we get that for any $\epsilon>0$ exists an $n_{2} \in N$, that for every $\lambda>\mathrm{n}_{2}>\mathrm{n}_{1}$ there exists an $\mathrm{m}: 1 \leq \mathrm{m} \leq \mathrm{k}_{2}-\mathrm{k}$ $1-2$, that performed the inequalities

$$
Q_{\lambda}^{*}(f,[a, b], \varepsilon) \leq Q_{\lambda}^{* *}(f,[a, b], \varepsilon)<\epsilon
$$

Now Theorem 2.1 follows from Proposition 2.5.

To prove the theorem 2.1 if $\mathrm{f} \in \mathrm{C}\left(\omega^{r} 1[\mathrm{a}, \mathrm{b}]\right) \cap$ $V^{+}(\mathrm{v})$ is sufficient to note that if $\mathrm{f} \in \mathrm{C}\left(\omega^{r} 1[\mathrm{a}, \mathrm{b}]\right) \cap$ $V^{+}(\mathrm{v})$, then $-\mathrm{f} \in \mathrm{C}\left(\omega^{l} 1[\mathrm{a}, \mathrm{b}]\right) \cap V^{-}(\mathrm{v})$ and operator $\mathrm{C}_{\Omega}$ (f, . ) - linear. Theorem 2.1 proved.

Remark 2.7. In the case when $f \in C\left(\omega^{l} 1[a, b]\right) \cap$ $\mathrm{V}$ (v) or $\mathrm{f} \in \mathrm{C}\left(\omega^{\mathrm{r}} 1[\mathrm{a}, \mathrm{b}]\right) \cap \mathrm{V}$ (v) ( $\mathrm{v}$ is the majorant classic module change $v(n, f))$ in $^{[33]}$ proved that the conditions of the form (2.2) are sufficient for the uniform convergence of trigonometric interpolation processes and sequences of classical Lagrange interpolation polynomials with the matrix of interpolation nodes P.L. Chebyshev.

The paper ${ }^{[34]}$ set uniform convergence of trigonometric Fourier series for the $2 \pi$-periodic, functions of the class $\mathrm{f} \in \mathrm{C}(\omega \quad 1[\mathrm{a}, \mathrm{b}]) \cap \mathrm{V}(\mathrm{v}))$, where functions $\omega(f, \delta)$ are majorants classical modulus of continuity $\mathrm{w}(\mathrm{f}, \mathrm{b})$ and module changes $\mathrm{v}(\mathrm{n}, \mathrm{f})$.

Remark 2.8. From Theorem 2.1 it follows that if $\mathrm{f}_{1}$ $\in \mathrm{C}\left(\omega_{1}^{\mathrm{r}}[[\mathrm{a}, \mathrm{b}]) \cap \mathrm{V}^{+}\left(\mathrm{v}_{1}\right)\right.$, and $\mathrm{f}_{2} \in \mathrm{C}\left(\omega_{2}^{\mathrm{r}}[[\mathrm{a}, \mathrm{b}]) \cap\right.$ $\mathrm{V}^{-}\left(\mathrm{v}_{2}\right)$, and the two pairs of functions $\left(\mathrm{v}_{\mathrm{i}}, \omega_{\mathrm{i}}\right)$, where $\mathrm{i}=1$, 2 , satisfy the relation (2.2), that, although a linear combination of $f=a f_{1}+\beta f_{2}$ can non-belong to any of classes, however because of the linearity of the operator $\mathrm{C}_{\Omega}(\mathrm{f}, \cdot)$, will have the relate $(2.3)$.
Remark 2.9. Each of the classes of functions: Dini-Lipschitz $\lim _{n \rightarrow \infty} \omega\left(\mathrm{f}, \frac{1}{\mathrm{n}}\right) \ln \mathrm{n}=0 \quad$ (see $^{[20]}$, Corollary 2), and satisfying the condition of Krylov (continuous function of bounded variation), is a subset of functional class, described by the terms (2.2).

Remark 2.10. If $\mathrm{f} \in \mathrm{C}[0, \pi]$ there are the relations $v^{+}(n, f) \leq v(n, f) \leq 2\left(v+(\mathrm{n}, f)+\|f\|_{C[0, \pi]}\right.$, $-v^{-}(n, f) \leq v(n, f) \leq 2\left(-v^{-}(n, f)+\|f\|_{C[0, \pi]}\right.$,

Corollary 2.11. From Theorem 2.1 follow that $\lim _{n \rightarrow \infty} \omega^{l}\left(f, \frac{1}{n}\right) \ln n=0 \quad$ or $\lim _{n \rightarrow \infty} \omega^{r}\left(f, \frac{1}{n}\right) \ln n=0$ ensure fairness (2.3).

Corollary 2.12. If a non-decreasing, concave function of natural argument $\mathrm{v}$ such that

$$
\sum_{k=1}^{\infty} \frac{v(k)}{k^{2}}<\infty
$$

then for any function $f \in C[0, \pi] \cap \mathrm{V}^{ \pm}$(v)is true ratio (2.3)

Proof. Indeed, from the continuity of $f$ implies the existence of a sequence of positive integers $\left\{m_{n}\right\}_{n=1}^{\infty}$ such that $\lim _{n \rightarrow \infty} m_{n}=\infty$ to and $\lim _{n \rightarrow \infty} \omega\left(f, \frac{1}{n}\right) \ln \quad m_{n}=0$. Therefore, the convergence of series (2.32) ensures that the condition (2.2) for any function $f$, belonging to at one the classes of $C[0, \pi]$ $\cap \mathrm{V}^{+}(\mathrm{v})$ or $\mathrm{C}[0, \pi] \cap \mathrm{V}^{-}(\mathrm{v})$. The proof is complete.

\section{References}

1. Stenger F. Numerical Metods Based on Sinc and Analytic Functions, N.Y., Springer Ser. Comput. Math. 1993; 20 Springer-Verlag.

2. Livne Oren E., Brandt Achi E. MuST: The multilevel sinc transform, SIAM J. on Scientific Computing, 2011; 33(4): 1726-1738.

3. Coroianu L, Sorin G. Gal Localization results for the non-truncated max-product sampling tors based on Fejer and sinc-type kernels, Demonstratio Mathematica 2016; 49(1): 38-49.

4. Richardson M., Trefethen L. A sinc function analogue of Chebfun, SIAM J. SCI. COMPUT. 2011; 33(5): 25192535.

5. Khosrow M., Yaser R., Hamed S. Numerical Solution for First Kind Fredholm Integral Equations by Using Sinc Collocation Method, International Journal of Applied Physics and Mathematics. 2016; 6(3): 120-128.

6. Marwa M. Tharwat Sinc approximation of eigenvalues of Sturm - Liouville problems with a Gaussian multiplier Calcolo: a quarterly on numerical analysis and theory of computation 2014; 51(3): 465-484.

7. Alquran MT., Al-Khaled K. Numerical Comparison of Methods for Solving Systems of Conservation Laws of Mixed Type, Int. Journal of Math. Analysis 
2011; 5(1): 35 - 47.

8. Chen J., Lu E., Huang B. Sampling theorem for multiwavelet subspaces / J. Chen, E. Lu, B. Huang // Journal of Shanghai University (English Edition) . 2007; 11(6): 570575.

9. Daubechies I. Ten Lectures on Wavelets, Socieety for industrial and appled Mathematics, Philadelphia, Pennsylvania 1992.

10. Ya NI., Stechkin SB. Basic constructions of wavelets, Fundam. Prikl. Mat. 1997; 3(4): 999-1028 English version: Russian Mathematical Surveys 1998; 53(6): 1159-1231.

11. Maksimenko IE, Skopina MA. Multivariate periodic wavelets, St. Petersburg Math. J. 2004; 15(2): 165-190 English version: St. Petersburg Mathematical Journal 2004; 15(2): 165-190.

12. Brown JLJ. On the error in reconstructing a nonband, limited function by means of bandpass sampling theorem, J. of Mathematical Analysis and Applications 1967; 18: 75-84.

13. Butzer PL, Higgins JR, Stens RL. Classical and approximate sampling theorems: studies in the $\mathrm{Lp}(\mathrm{R})$ and the uniform norm, Journal of Approximation Theory 2005; 137: 250-263.

14. Shmukler AI, Shulman TA. Certain properties of Kotel'nikov series, Izv. Vyssh. Uchebn. Zaved. Mat. 1974; 3: 93-103.

15. Sklyarov VP. On the best uniform sinc-approximation on a finite interval, East Journal on Approximations 2008; 14: 183-192.

16. Zayed AI, Schmeisser G. (eds.) New Perspectives on Approximation and Sampling Theory, Applied and Numerical Harmonic Analysis /A.I. Zayed, G. Schmeisser // Springer International Publishing Switzerland 2014.

17. Kivinukk A., Tamberg G. Interpolating generalized Shannon sampling operators, their norms and approximation properties, Sampl. Theory Signal Image Process. 2009; 8: 77-95.

18. Trynin AY, Sklyarov VP. Error of sinc approximation of analytic functions on an interval, Sampling Theory in Signal and Image Processing 2008; 7(3): 263-270.

19. Trynin AY. Estimates for the Lebesgue functions and the Nevai formula for the sinc-approximations of continuous functions on an interval, Sibirsk. Mat. Zh. 2007; 48(5): 1155-1166. English transl. Siberian Mathematical Journal September 2007; 48(5): 929-938.

20. Trynin AY. Tests for pointwise and uniform convergence of sinc approximations of continuous functions on a closed interval, Mat. Sb. 2007; 198(10): 141-158. English transl. Sbornik: Mathematics 2007; 198(10): 1517-1534.

21. Trynin AY. A criterion for the uniform convergence of sinc-approximations on a segment, Izv. Vyssh. Uchebn. Zaved. Mat. 2008; 6: 66-78. English transl.Russian Mathematics June 2008; 52(6): 58-69.
22. Trynin A.Yu. On divergence of sinc-approximations everywhere on (0, n), Algebra i Analiz 2010; 22(4): 232-256. English transl. St. Petersburg Math. J. 2011; 22: 683-701.

23. Trynin AY. A generalization of the Whittaker-Kotel'nikov-Shannon sampling theorem for continuous functions on a closed interval, Mat. Sb. 2009; 200(11): 61-108. English version: Sbornik: Mathematics. 2009 200(11): 1633-1679.

24. Trynin AY. On the absence of stability of interpolation in eigenfunctions of the Sturm-Liouville problem, Izv. Vyssh. Uchebn. Zaved. Mat. 2000; 9: 60-73. English version: Russian Mathematics (Izvestiya VUZ. Matematika) 2000; 44(9): 58-71.

25. Trynin AY. The divergence of Lagrange interpolation processes in eigenfunctions of the Sturm-Liouville problem, Izv. Vyssh. Uchebn. Zaved. Mat. 2010; 11: 74-85. English version: Russian Mathematics (Izvestiya VUZ. Matematika) 2010; 54(11): 66-76.

26. Trynin AY. On operators of interpolation with respect to solutions of a Cauchy problem and Lagrange-Jacobipolynomials, Izv. RAN. Ser. Mat. 2011; 75(6): 129-162. English version: Izvestiya: Mathematics. 2011; 75(6): 1215-1248.

27. Trynin AY. On some properties of sinc approximations of continuous functions on the interval, Ufimsk. Mat. Zh. 2015; 7(4): 116-132. English version: Ufa Mathematical Journal. 2015; 7(4): 111-126. 10.13108/2015-7-4-111

28. Trynin A.Yu. On necessary and sufficient conditions for convergence of sinc approximations, Algebra i Analiz 2015; 27(5): 170-194.

29. Trynin AY. Approximation of continuous on a segment functions with the help of linear-combinations of sincs, Izv. Vyssh. Uchebn. Zaved. Mat. 2016; 3: 72-81. English version: Russian Mathematics (Izvestiya VUZ. Matematika) 2016; 60(3): 63-71.

30. Trynin AY. On inverse nodal problem for Sturm-Liouville operator, Ufimsk. Mat. Zh. 2013; 5(4): 116-129. English version: Ufa Mathematical Journal. 2013; 5(4): 112-124. 10.13108/2013-5-4-112

31. Trynin AY. Differential properties of zeros of eigenfunctions of the Sturm-Liouville problem, Ufimsk. Mat. Zh. 2011; 3(4): 133-143.

32. Mochizuki K, Trooshin IY. Evolution equations of hyperbolic and Schrodinger type. Asymptotics, estimates and nonlinearities. Based on a workshop on asymptotic properties of solutions to hyperbolic equations, London, UK. 2011; 227-245.

33. Privalov AA. Uniform convergence of Lagrange interpolation processes, Math. Notes. 1986; 39(2): 124-133. English version: Mathematical Notes 1986; 39(2): 124-133.

34. Chanturiya ZA. On uniform convergence of Fourier series, Math. USSR-Sb. 1976; 29(4): 475-495.

35. Dyachenko MI. On a class of summability methods for multiple Fourier series, Mat. Sb. 2013; 204(3): 
3-18. English version: Sbornik: Mathematics. 2013; 204(3): 307-322.

36. Volosivets SS, Golubov BI. Uniform Convergence and Integrability of Multiplicative Fourier Transforms, Mat. Zametki. 2015; 98(1): 44-60. English version: Mathematical Notes. 2015; 98(1): 53-67.

37. Farkov YA. On the best linear approximation of holomorphic functions, Fundam. Prikl. Mat. 2014; 19(5): 185-212.

38. Borisov DI., Dmitriev SV. On the spectral stability of kinks in 2D Klein-Gordon model with pari- ty-time-symmetric perturbation, Studies in Applied Mathematics. 2017; 138(3): 317-342.

39. Borisov D, Cardone G, Durante T. Homogenization and norm resolvent convergence for elliptic operators in a strip perforated along a curve, Proceedings of the Royal Society of Edinburgh, Section: A Mathematics 2016; 146(6): 1115 - 1158.

40. Pokornyi YV., Zvereva MB., Shabrov SA. Sturm-Liouville oscillation theory for impulsive problems. Uspekhi Mat. Nauk. 2008; 63:1(379) $111-154$ 